\title{
La nueva reforma del mercado eléctrico español: eficiencia y austeridad*
}

\author{
Aitor Ciarreta Antuñano \\ Cristina Pizarro-Irizar \\ Universidad del País Vasco/Euskal Herriko Unibertsitatea \\ (UPV/EHU), BRIDGE
}

\section{Resumen}

El año 2014 está siendo marcado por intensos cambios regulatorios en el ámbito eléctrico, tras la aprobación de la nueva Ley del Sector Eléctrico. Entre otras medidas, se abre la puerta a un nuevo sistema de tarificación a consumidores finales y se reforma el apoyo a la generación mediante fuentes renovables. Estas iniciativas buscan incrementar la eficiencia del sistema en un contexto de austeridad presupuestaria amenazado por un desbocado déficit tarifario. Aún es pronto para evaluar su efectividad, pero ciertos indicadores señalan que todavía peligran los objetivos marcados y que medidas complementarias serían necesarias para lograr un mercado efectivamente competitivo.

Palabras clave: mercado eléctrico, energía renovable, déficit tarifario.

Clasificación JEL: L51, Q4.

\begin{abstract}
The year 2014 is being marked by intense regulatory changes in the electricity sector, after the adoption of the new Electricity Act. Among other measures, it opens a door to a new pricing system for last resort consumers and it also reforms the support system for renewable generation. These initiatives seek to increase the efficiency of the system in a context of budgetary austerity threatened by an increasing tariff deficit. It is too early to assess its effectiveness, but certain indicators show that some objectives are jeopardized and further measures would be needed to achieve a really competitive market.
\end{abstract}

Keywords: electricity market, renewable energy, deficit of regulated activities.

JEL classification: L51, Q4.

* Los autores agradecen la financiación del Ministerio de Economía y Competitividad, ECO2012-35820, y del Departamento de Educación. Universidades e Investigación del Gobierno Vasco, IT-783-13. Cristina Pizarro-Irizar agradece al Gobierno Vasco la financiación recibida a través del Programa de Ayudas para la Formación y Perfeccionamiento del Personal Investigador del Departamento de Educación, Universidades e Investigación (BFI-2011-301), así como la financiación del programa de Ayudas a la Investigación en Energía y Medio Ambiente «Energía para la investigación» de la Fundación Iberdrola. 


\section{Introducción}

La regulación en las actividades de generación y comercialización de los mercados mayoristas y minoristas sometidos a una regulación específica, pero donde los precios se fijan en condiciones de competencia, debe buscar la eficiencia. La evolución del mercado eléctrico español en los últimos años ha estado marcada por tres hechos fundamentales que han obligado a una profunda revisión del marco regulatorio existente. En primer lugar, el fuerte crecimiento en la generación mediante fuentes de energía renovables a partir de la aprobación del RD 661/2007, de 25 de mayo. En segundo lugar, la supresión en diciembre de 2013 de las subastas CESUR (Contratos de Energía para el Suministro de Último Recurso) para el suministro eléctrico a consumidores regulados. En tercer lugar, y relacionado con los cambios anteriores, el crecimiento desbocado del déficit de tarifa de las actividades reguladas. Así pues, los desajustes se han ido agravando tanto en el lado de la demanda como en el de la oferta del mercado.

La garantía de suministro también juega un papel importante en la eficiencia del sistema. Ésta consiste precisamente en ofrecer capacidad suficiente para poder abastecer la máxima demanda horaria producida (indicador conocido como índice de cobertura), y se reduce sustancialmente con la presencia de energía renovable ${ }^{1}$, dado su carácter intermitente y no gestionable. Sin embargo, a pesar de la elevada participación de tecnologías renovables en el mix eléctrico actual, la evidencia empírica demuestra que el índice de cobertura en España ${ }^{2}$ es siempre superior al valor de referencia, garantizando el margen de seguridad mínimo exigido del 10 por 100 y dejando claro que el sistema español tiene una notable sobrecapacidad. La capacidad de refuerzo es efectivamente necesaria y puede lograrse a través de mecanismos como los pagos por capacidad, por ejemplo; pero el hecho de presentar sobrecapacidad reduce considerablemente la eficiencia del sistema, dados los costes que su mantenimiento conlleva para el reducido uso que se realiza de ella (aún menor en un contexto de demanda reducida como el que se vive actualmente). De hecho, según publica el operador del mercado eléctrico en sus informes anuales, el papel de los pagos por capacidad en el precio final de la electricidad se ha elevado de un 1,62 por 100 que representaba en 2008 a un 10,40 por 100 que suponía ya a cierre de 2013, denotando nuevamente la sobrecapacidad del sistema y los costes que ello supone.

Junto a la eficiencia en la asignación de los recursos, otro de los objetivos del diseño del mercado eléctrico debería ser la minimización del riesgo regulatorio. Éste se asocia a la credibilidad de los compromisos regulatorios adquiridos y a la no

\footnotetext{
${ }^{1}$ La baja interconexión del mercado eléctrico español con sus países vecinos también influye en la garantía de suministro.

2 Según los valores calculados en CIARRETA et al. (2014), el índice de cobertura sin renovables para 2008 era de 1,17 , para 2009 de 1,13 , para 2010 de 1,20 , para 2011 de 1,18 y para 2012 de 1,15 . Incluso sin considerar la energía renovable, en todos los casos la cobertura se encuentra por encima del valor de referencia 1,1, marcado por el operador del sistema eléctrico como valor mínimo de margen de seguridad.
} 
existencia de comportamientos oportunistas por parte del regulador. Los frecuentes cambios regulatorios han creado una sensación de inseguridad entre los inversores, no solamente porque alteran cada poco tiempo las reglas de un mercado caracterizado por inversiones a largo plazo, sino porque con estas medidas no está claro que se atajen los problemas que subyacen al sector.

En 2014, el marco regulatorio ha sufrido un cambio espectacular en todas sus vertientes. Por un lado, desde la perspectiva de la demanda, se han cancelado las subastas CESUR y han sido reemplazadas por un nuevo sistema para el cálculo de la factura eléctrica, en el que el objetivo es que el precio refleje realmente los costes de producción. Por otro lado, afectando a la oferta, con la aprobación del RD-1 1/2012 en 2012 y de los RD-1 2/2013 y RD-1 9/2013 en 2013, se ha ido eliminando de forma paulatina el sistema de primas y tarifas que venía aplicándose al llamado Régimen Especial $^{3}$ (RE) desde 2004, supresión consolidada en junio de 2014 (de forma retroactiva) con el RD 413/2014, de 6 de junio. Los incentivos a las renovables han cambiado buscando garantizar una rentabilidad «normal» de las unidades existentes y se ha eliminado el RE como tal. El argumento es el siguiente: en la medida en que los costes de inversión del RE se han reducido de manera sustancial, si la escala de operación es suficiente, ya resultaría factible que se alcanzaran niveles de costes normales de producción.

Con estas medidas, el organismo regulatorio persigue dos objetivos fundamentales: (i) controlar y eventualmente eliminar el déficit tarifario que genera, entre otras, fuertes distorsiones en las decisiones de inversión de los agentes, y (ii) que el mecanismo de precios refleje realmente los costes de producción. Esto es, una reforma que impulse la eficiencia, condicionada lo menos posible por la austeridad necesaria dadas las restricciones presupuestarias y financieras actuales.

El trabajo se estructura de la siguiente manera. En la sección 2 hacemos un repaso al marco regulatorio anterior al aprobado en 2014 y analizamos algunas de las distorsiones que ha creado. En la sección 3 analizamos el nuevo marco regulatorio. La sección 4 continúa con una breve discusión sobre las perspectivas que hay para control del déficit y la creación de un mercado eficiente. Por último, se recogen los puntos más relevantes de la reforma y perspectivas a corto plazo en las conclusiones.

\section{Marco regulatorio anterior a la reforma}

Tres elementos del marco regulatorio han determinado en buena medida el comportamiento de los agentes del mercado. En primer lugar, las subastas CESUR de suministro eléctrico a los consumidores de último recurso. En segundo lugar, el

\footnotetext{
${ }^{3}$ Dentro del Régimen Especial se incluyen las tecnologías renovables (solar fotovoltaica, termosolar, eólica, hidráulica de menos de $50 \mathrm{MW}$ o mini-hidráulica, biomasa, residuos y tratamiento de residuos) y la cogeneración.
} 
fuerte crecimiento de la capacidad instalada de energías renovables. Finalmente, y relacionado con lo anterior, el fuerte crecimiento del déficit tarifario y las importantes dificultades para su financiación.

\subsection{Subastas CESUR}

En los años 2005 y 2006 el fuerte crecimiento del precio del pool (más de un 50 por 100 con respecto a 2004) empujó el paso de los consumidores del mercado libre a la tarifa regulada, ya que ésta se encontraba muy por debajo del precio del mercado libre. De esta forma, se hacía imposible que las empresas comercializadoras pudiesen competir en condiciones de igualdad con las distribuidoras, lo que agravó el aún incipiente déficit de tarifa (ver Gráfico 5). Si bien es cierto que este paso a tarifa regulada era el resultado lógico de decisiones de consumidores racionales, sí que desvirtuaba el objetivo fundamental de un mercado competitivo establecido en la Directiva 2003/54/CE. Así pues, la reforma buscaba diseñar un sistema de fijación de precios que reflejara los costes reales de suministro de electricidad, de tal forma que contuviera el déficit tarifario y fomentara la competencia entre proveedores finales ${ }^{4}$.

Las subastas CESUR eran un mecanismo destinado a la compra de energía para aquellos consumidores que habían optado por la tarifa regulada denominada Tarifa de Último Recurso (TUR) $)^{5}$. Su implementación mediante la Orden ITC/400/2007 creaba un régimen de subastas trimestrales para los consumidores de último recurso paralelo al del mercado diario para el suministro de electricidad. Este método buscaba fomentar la liquidez del mercado a plazo y estabilizar el precio de la TUR, estableciendo un precio más cercano a la realidad, de tal forma que se redujera en lo posible el déficit tarifario. La implantación de las subastas permitía en principio obtener un precio competitivo para la electricidad, donde los agentes se comprometían, por medio de un contrato bilateral, a suministrar energía al precio fijado en la subasta. Con este precio, se evitaba la aparición de diferencias entre coste de la electricidad previsto por el regulador al principio de año y el precio de mercado.

En principio existían dos mecanismos que en cierta medida buscaban racionalizar el consumo aumentando la elasticidad de la demanda inter-horaria (disponibilidad para elegir entre tarifa con discriminación horaria y tarifa sin discriminación horaria) y cumplir con las nuevas directrices europeas (Directiva 72/2009) eliminando restricciones, lo que abarataba costes de búsqueda y cambio de suministrador. Cuando

4 Con anterioridad a la implantación de las subastas CESUR fue aprobado el Real Decreto-ley 3/2006, de 24 de febrero, que modificaba el mecanismo de casación de las ofertas de venta y adquisición de energía presentadas por empresas verticalmente integradas y que suponía la eliminación práctica del mecanismo de mercado en la fijación de precios. Este sistema fue eliminado en febrero de 2007.

${ }^{5} \mathrm{El}$ principio que inspiraba este tipo de subastas era proteger al pequeño consumidor de la excesiva volatilidad del mercado libre. A la TUR se podían acoger entes con una potencia contratada igual o menor a $10 \mathrm{KW}$. 
el consumidor optaba por acogerse a la TUR, podía solicitar en cualquier momento el cambio de una entidad comercializadora a otra o incluso pasarse al mercado libre. Para ello, era necesario comunicárselo al nuevo comercializador y firmar un nuevo contrato encargándose dicha empresa de todos los trámites necesarios. Una vez que el cambio se había efectuado, durante un año las empresas competidoras no podían realizar contraofertas para captar al cliente. La denominada Oficina de Cambios de Suministrador (OCSUM) era la responsable de la supervisión de los cambios de suministrador, «conforme a los principios de transparencia, objetividad e independencia». En su capital participaban obligatoriamente los distribuidores y comercializadores de electricidad.

Estas subastas asignaban contratos mediante pujas descendientes en la que los agentes ofertaban en diferentes rondas un precio del suministro eléctrico, disminuyendo en cada una el precio hasta que la cantidad ofertada fuera igual o inferior a la demandada ${ }^{6}$. En el Cuadro 1 se reportan varios momentos de la distribución de los resultados de las diferentes subastas que se han realizado entre 2009 y 2013. Entre otros, se presentan número de participantes, número de rondas realizadas en esa subasta, participantes cualificados, cantidad adjudicada, cantidad punta trimestral, precio base y precio punta ${ }^{7}$.

\section{CUADRO 1}

\section{DESCRIPTIVOS SUBASTAS CESUR}

\begin{tabular}{|c|c|c|c|c|c|c|}
\hline & \multirow{2}{*}{$\begin{array}{c}\text { N. }{ }^{\circ} \text { de } \\
\text { partici- } \\
\text { pantes }\end{array}$} & \multirow{2}{*}{$\begin{array}{l}\mathrm{N}^{\circ} \text { de } \\
\text { rondas }\end{array}$} & \multicolumn{2}{|c|}{ Cantidad } & \multicolumn{2}{|c|}{ Precio } \\
\hline & & & Base & Punta & Base & Punta \\
\hline Media & 30 & 15 & 3.605 & 464 & 48,55 & 53,99 \\
\hline Mínimo & 23 & 7 & 2.400 & 200 & 36,58 & 38,22 \\
\hline Máximo & 37 & 22 & 5.000 & 688 & 58,06 & 66,84 \\
\hline Desv. estándar & 3,294 & 3,183 & 848,2 & 136,3 & 6,15 & 6,90 \\
\hline Apuntamiento & 0,009 & $-0,884$ & 0,129 & 0,094 & $-0,14$ & $-0,40$ \\
\hline Curtosis & 2,944 & 4,437 & 1,835 & 2,11 & 2,28 & 3,01 \\
\hline
\end{tabular}

FUENTE: Elaboración propia a partir de datos del Operador del Mercado Ibérico de Electricidad (OMIE).

Tanto la distribución del número de participantes como de precios punta es aproximadamente normal, mientras que el exceso de curtosis es únicamente positivo para el número de rondas. Esto nos da una idea de que tanto los precios como las cantidades tienen distribuciones platicúrticas y ligeramente asimétricas, lo cual implica que las colas de la distribución empírica de los precios son más anchas que

\footnotetext{
${ }^{6}$ Una descripción completa del funcionamiento de las subastas CESUR se puede encontrar en http:// www.subastascesur.omie.es/subastas-cesur/.

7 Se consideran horas de carga base todas las horas del día y horas de carga punta de 8:00 a 20:00, de lunes a viernes.
} 
una normal y que se produce una mayor dispersión en los precios bajos que en los altos.

A modo de comparación, los Gráficos 1 y 2 representan los precios medios base y punta respectivamente para cada subasta frente al precio medio trimestral del mercado diario resultante del plan diario básico de casación (PDBC).

Durante el período de vigencia de las subastas, la diferencia media en carga base entre el precio CESUR y el PDBC fue de 4,55 euros por MWh. Esta variación estaría incluyendo la prima de riesgo habitual de un contrato a plazo (tres meses en este caso). Lo que sí resulta significativo es cómo en las subastas 9 y 10, para suministro del cuarto trimestre de 2009 y del primer trimestre de 2010, la diferencia de precios fue de 12,08 y 14,05 euros por MWh, respectivamente. Asimismo, para las subastas 21 y 22, para suministro del primer y segundo trimestre de 2013, la diferencia fue de 13,84 y 11,15 euros por MWh, para posteriormente caer a $-4,85$ euros por MWh en la subasta 24 , lo que implicaría un importante error de predicción por parte de los agentes vendedores. Finalmente, es de destacar la reducción progresiva en los volúmenes de subasta de producto base.

\section{GRÁFICO 1}

PRECIOS BASE (€/MWh) CESUR Y MERCADO DIARIO

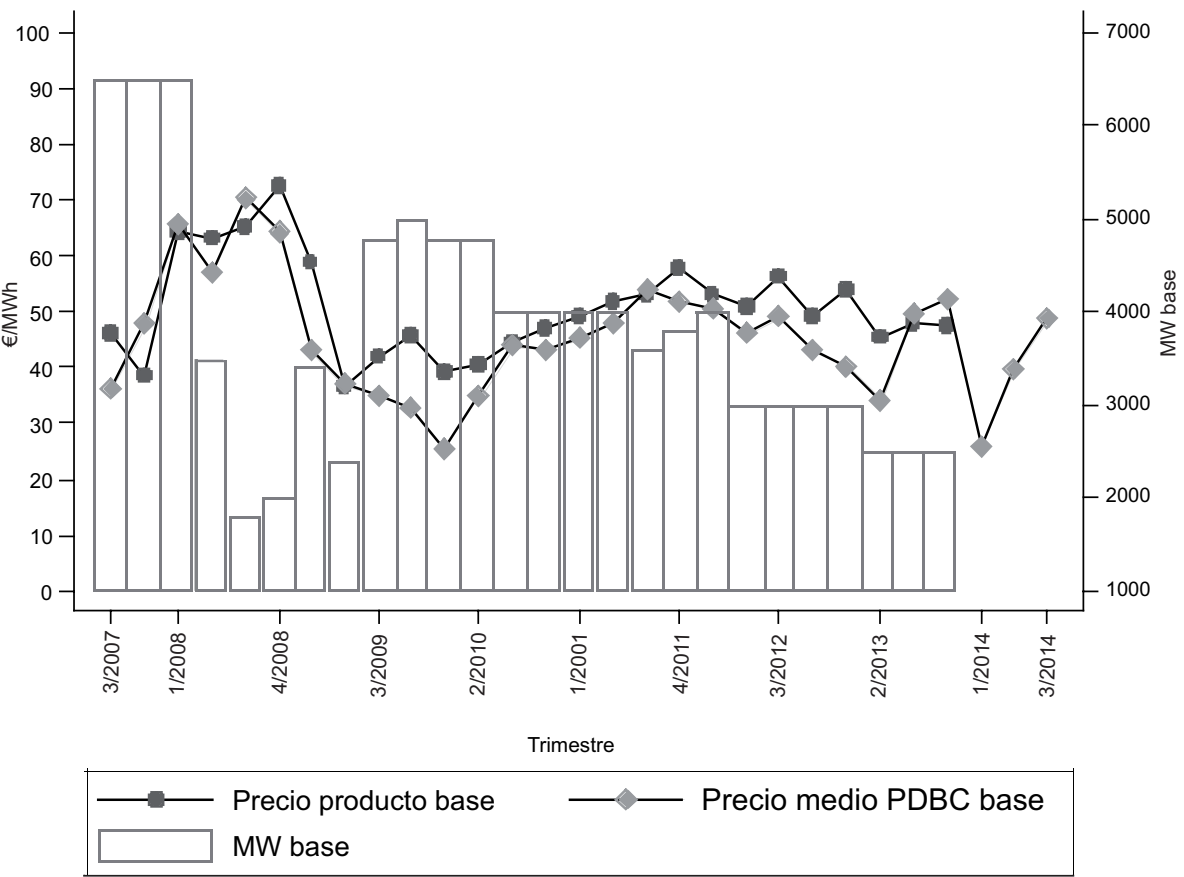

FUENTE: Elaboración propia a partir de datos de OMIE. 


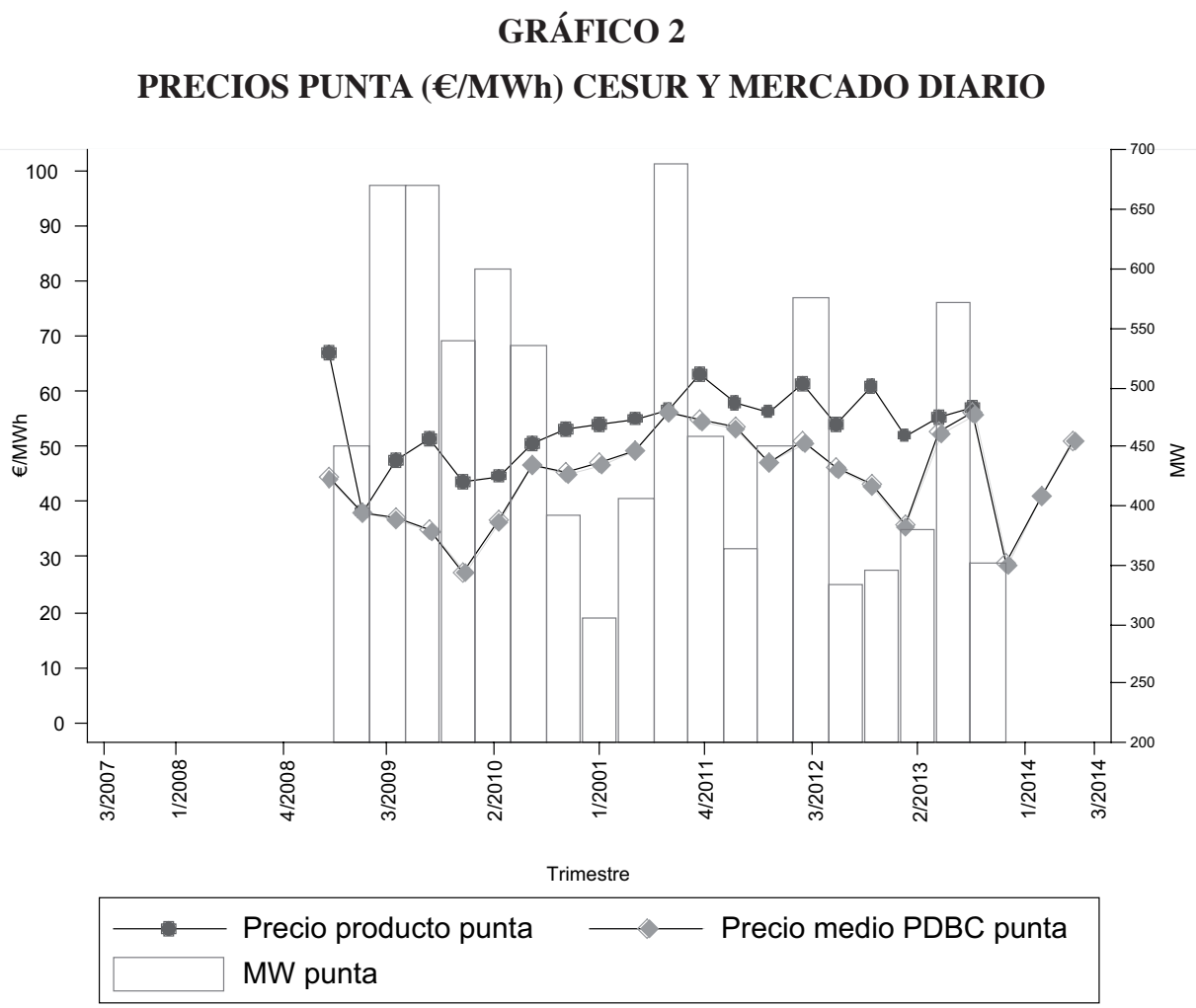

FUENTE: Elaboración propia a partir de datos de OMIE.

En carga punta, la diferencia media entre el precio CESUR y el PDBC fue de 8,81 euros por MWh, no existiendo ningún trimestre con variaciones de precios negativas. Como es de esperar, ambas diferencias de precios están correlacionadas positivamente. Así pues, existe correlación positiva y significativa entre los precios CESUR y los precios del mercado diario, más fuerte en horas punta.

El resultado de la 25 subasta CESUR de suministro a los consumidores TUR de diciembre de 2013 supuso un elemento más a añadir en la crisis del sector eléctrico. Como resultado, la Comisión Nacional de los Mercados y la Competencia (CNMC), en virtud de las facultades que tenía asignadas para verificar el normal funcionamiento de la subasta, abrió una investigación a instancia del Ministerio de Industria, Energía y Turismo sobre «los movimientos inusuales en los precios mayoristas de la electricidad». OMIE tenía la facultad de suspender la subasta si existiesen argumentos que indicasen «insuficiente presión competitiva» ${ }^{8}$.

\footnotetext{
${ }^{8}$ Así quedaba establecido en el criterio 9 del Anexo de la Orden ITC/1601/2010, de 11 de junio. Esta misma orden concretaba la función supervisora de la CNMC, junto con la Orden ITC/1659/2009, de 22 de junio.
} 
Los precios en el mercado diario venían registrando un repunte poco habitual en el mes de diciembre (precio medio de 80,41 euros por MWh) muy por encima de la media de noviembre (41,81 euros el $\mathrm{MWh}$ ) o de octubre (51,50 euros el $\mathrm{MWh}$ ). Entre los factores objetivos que favorecieron el incremento de precios estaban las bajas temperaturas y baja generación eólica, pero los picos de precios en el mercado diario superaban los 100 euros por MWh. Así resultaba alarmante cómo durante el período de vigencia de las subastas CESUR sólo había habido 57 precios horarios por encima de 100 euros por MWh, de los cuales 50 se habían dado en diciembre de $2013^{9}$.

A continuación analizamos los desajustes señalados por la CNMC que atañen directamente a la gestión de los mercados diario e intradiario y fueron juzgados suficientes para cancelar la subasta.

\section{Desajustes en el mercado diario}

El fuerte incremento puntual de la demanda por motivos climatológicos en un contexto de caída generalizada por la bajada en la actividad económica impulsó al alza los precios de mercado. El Gráfico 3 representa tanto la tasa de crecimiento de la demanda intermensual como interanual durante el período de vigencia de las subastas.

\section{GRÁFICO 3}

\section{TASA DE CRECIMIENTO DE LA DEMANDA}

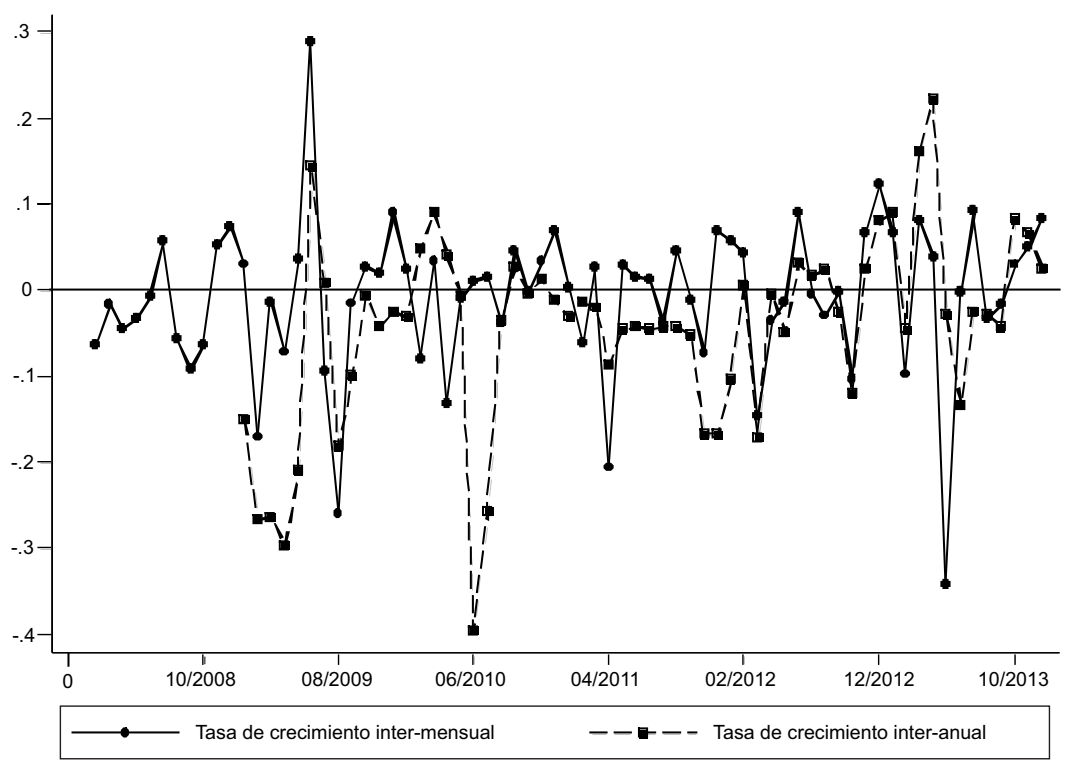

FUENTE: Elaboración propia a partir de datos de Red Eléctrica de España (REE).

9 La CNMC ya tenía abierto un expediente investigador de este incremento de precios con anterioridad al de la cancelación de la 25 subasta CESUR. 
Si bien es cierto que se produjo un repunte en la demanda tanto intermensual como interanual en diciembre de 2013, éste forma parte de un último trimestre de repunte en la demanda en general durante el período de vigencia de las subastas, las horas de máxima demanda se dieron durante varios días de junio de 2009. Por lo tanto, no parece ser la demanda el único factor determinante en el fuerte incremento de los precios.

Por el lado de la oferta se produjo un fuerte incremento de indisponibilidad de tecnologías de generación de carga base: nuclear y renovables. La disponibilidad de las plantas tiene una componente aleatoria que no es posible prever. Otro tema es la disponibilidad estratégica de parte de los generadores para influir sobre los precios finales (capacity withholding), que la CNMC no llega a señalar como causa determinante.

\section{Desajustes en el mercado intradiario}

La participación de los agentes en el mercado intradiario depende en principio de la necesidad de ajuste que tengan con respecto al programa del mercado diario. La fuerte penetración de las energías renovables, especialmente la generación eólica, ha incrementado sensiblemente la importancia de este mercado. La prioridad de acceso a la red de este tipo de generación más intermitente ha hecho que el mercado diario sea más volátil, por ejemplo desde 2007 el volumen de transacciones del mercado diario ha pasado a representar del 90 por 100 del volumen total de electricidad negociada en 2007 al 83 por 100 en 2013, con un mínimo del 78 por 100 en 2012. De la misma forma, utilizando el coeficiente de variación como medida de volatilidad, éste ha pasado de 0,085 en 2007 a 0,116 en 2013 en el mercado diario, y de 0,167 en 2007 a 0,274 en 2013 en el mercado intradiario. El Gráfico 4 representa el volumen de transacciones en el mercado intradiario para el período MIBEL hasta septiembre de 2014.

La CNMC argumentaba que las compras en las distintas sesiones del mercado intradiario habían caído sensiblemente en diciembre con respecto a los dos meses anteriores. Así pues, es cierto que en comparación con 2011 y 2012, el volumen de electricidad negociado en las distintas sesiones del mercado intradiario fue claramente inferior en 2013 con dos picos bajos en abril y diciembre por causas similares. Pero la menor producción eólica (energía más intermitente) podría ser indicador de que había una menor necesidad de acudir al mercado intradiario por parte de los agentes para ajustar sus programas del mercado diario.

Por lo tanto, a partir de estos dos indicadores utilizados por la CNMC resulta difícil detectar un cambio en las estrategias de pujas por parte de los generadores que permitiera justificar el resultado de la 25 subasta CESUR. No está claro que la indisponibilidad de las plantas fuera estratégica, ni que el menor volumen de negocio en el mercado intradiario estuviera relacionado con un intento de mantener precios altos. Tampoco el argumento de las eléctricas del fuerte tirón de la demanda 


\section{GRÁFICO 4}

\section{CUOTA DEL MERCADO INTRADIARIO CON RESPECTO AL PDBC}

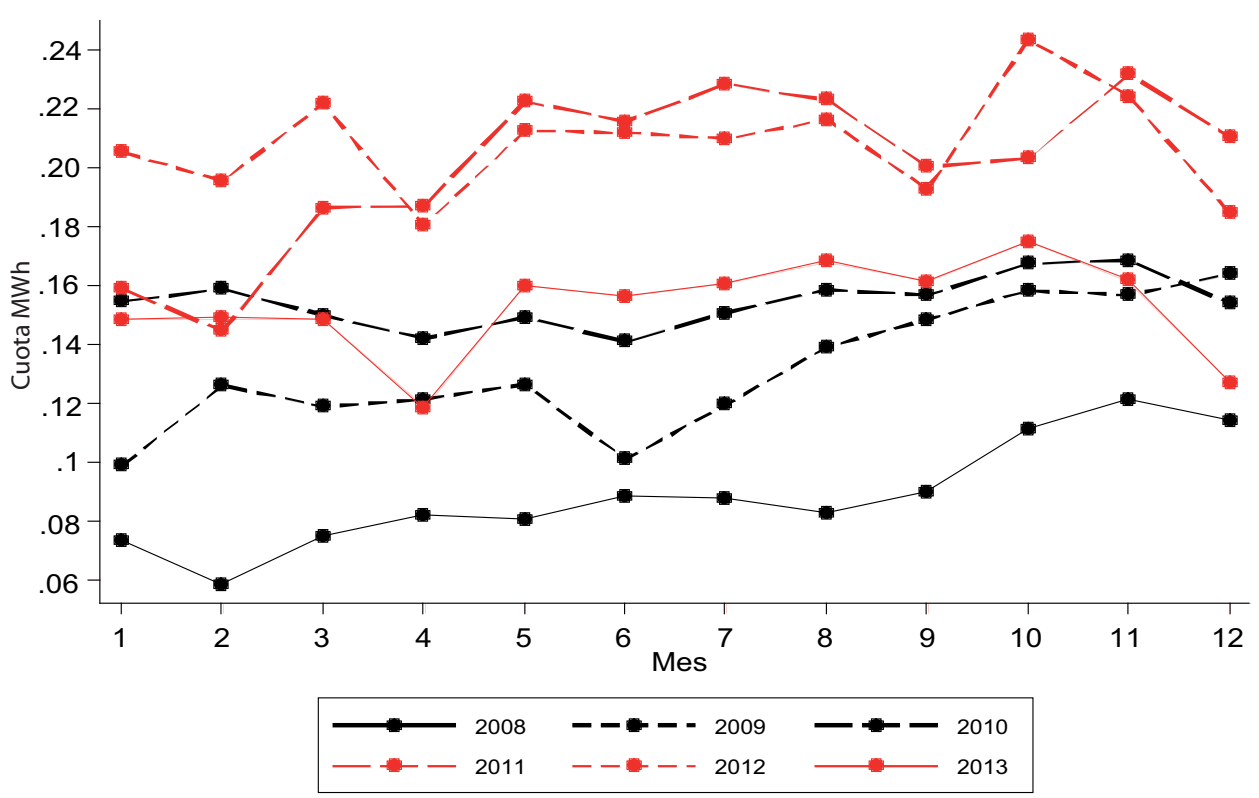

FUENTE: Elaboración propia a partir de datos de OMIE.

se sostiene como elemento determinante del aumento de precios. Por lo tanto, el resultado de la 25 subasta debió ser objeto de un análisis más exhaustivo por parte de la CNMC antes de recurrir a la cancelación, porque no se transmite a los agentes una señal correcta sobre la normal aplicación de las leyes de competencia. La intervención directa del Estado debe ser la última e inevitable instancia en caso de un mal funcionamiento de los mercados.

\subsection{Energías renovables}

El régimen regulatorio establecido en el RD 661/2007, de 25 de mayo, otorgaba prioridad de acceso a red a la energía proveniente del RE, al mismo tiempo que la remuneraba mediante un sistema de tarifas y primas diferenciado por tecnología, según la opción de financiación escogida. Los productores de energía renovable y cogeneración debían ofrecer su energía en el mercado diario a precio cero y como remuneración podían escoger entre recibir una tarifa fija o el precio de mercado más una prima, de menor importe que la tarifa fija y sujeta a unos límites inferiores y superiores. Tanto la tarifa como la prima se encontraban reguladas vía real decreto, sufriendo ambas modificaciones en sus valores a lo largo del tiempo. Los peajes de 
acceso aplicados a los consumidores finales eran la principal fuente de ingresos del sistema eléctrico para financiar estas primas y el resto de costes, partida que ha sido insuficiente para hacer frente a los crecientes costes del sistema de primas y que por ello ha tenido que ser reducida en los últimos años.

Aunque con anterioridad ya se introdujeron medidas limitadoras de incentivos (por ejemplo, establecimiento de un número máximo de horas de producción sujetas a recibir subvención o revisión a la baja de los niveles de incentivos), las más destacadas comenzaron a producirse en 2012, cuando se aprobó el RD-1 1/2012 (conocido como moratoria renovable), en el que se cancelaban los incentivos a nueva capacidad instalada de RE, manteniéndose en todo caso los compromisos existentes y, por tanto, sin afectar a la seguridad jurídica del sistema. Posteriormente, en 2013 se fue suprimiendo de forma paulatina el sistema de primas (RD-1 2/2013) y tarifas (RD-1 9/2013), hasta que en junio de 2014 se aprobó el RD 413/2014, en el que se establece con retroactividad un nuevo sistema de retribución basado en el principio de rentabilidad razonable de las instalaciones (lo trataremos con más detalle en la sección 3.2).

El Cuadro 2 muestra para todas las tecnologías del RE el porcentaje de energía acogida a cada una de las dos modalidades de pago vigentes hasta 2013. La evolución de esta variable durante los años 2009, 2011 y 2013 pone de manifiesto el efecto de las distintas medidas regulatorias en la decisión de adopción de sistema retributivo.

\section{CUADRO 2}

\section{ENERGÍA DEL RÉGIMEN ESPECIAL ACOGIDA A TARIFA O A PRIMA MÁS PRECIO DE MERCADO}

(En \%)

\begin{tabular}{|l|c|c|c|c|c|c|}
\hline \multirow{2}{*}{ Tecnología } & \multicolumn{2}{c|}{2009} & \multicolumn{2}{c|}{2011} & \multicolumn{2}{c|}{2013} \\
\cline { 2 - 7 } & Tarifa & $\begin{array}{c}\text { Prima+ } \\
\text { Mercado }\end{array}$ & Tarifa & $\begin{array}{c}\text { Prima+ } \\
\text { Mercado }\end{array}$ & Tarifa & $\begin{array}{c}\text { Prima+ } \\
\text { Mercado }\end{array}$ \\
\hline Cogeneración & 33 & 67 & 82 & 18 & 86 & 14 \\
\hline Fotovoltaica & 99 & 1 & 100 & 0 & 100 & 0 \\
\hline Termo-solar & - & - & 100 & 0 & 100 & 0 \\
\hline Viento & 7 & 93 & 21 & 77 & 100 & 0 \\
\hline Mini-hidroeléctrica & 30 & 66 & 40 & 50 & 98 & 0 \\
\hline Biomasa & 35 & 63 & 63 & 29 & 100 & 0 \\
\hline Residuos & 1 & 95 & 0,4 & 82 & 84 & 16 \\
\hline Tratamiento de residuos & 100 & 0 & 100 & 0 & 100 & 0 \\
\hline Total & 28 & 72 & 52 & 46 & 96 & 4 \\
\hline
\end{tabular}

NOTAS: En algunos casos el porcentaje de energía acogida a venta a tarifa o prima más precio mercado no suma el 100 por 100, ya que parte de la energía (aunque poca) se vendió en otros mercados. Datos de termosolar reportados a partir de 2010 .

FUENTE: Elaboración propia a partir de datos de la CNE/CNMC (2002-2013) para 2009, 2011 y 2013. 
De esta manera, observamos que durante el año 2009 la mayor parte de la energía de RE estaba acogida a la opción de venta en mercado más prima (72 por 100), mientras que en 2013 apenas un 4 por 100 recibía ese tipo de retribución, como resultado directo de la anulación de la modalidad en febrero de ese mismo año. Por otro lado, se aprecia también de forma implícita la diferencia de niveles de retribución entre modalidades y tecnologías, puesto que a ciertas fuentes (por ejemplo, fotovoltaica y termo-solar) siempre les compensó más acogerse a la opción de tarifa, dada la cuantía a la que estaba establecida, mientras que los generadores eólicos han participado tradicionalmente más de la opción prima más precio de mercado.

Adicionalmente, el Cuadro 3 recoge por tipo de tecnología la capacidad instalada, energía vendida en el mercado diario y la prima unitaria (FIT/FIP) cobrada en los años 2009, 2011 y 2013. El concepto de prima unitaria expresa el incentivo unitario medio pagado a cada tecnología. Se refiere al coste real por megavatio del sistema de incentivos y se calcula como el importe total anual destinado al pago de retribuciones, bien en forma de tarifas o bien en forma de primas, dividido por la energía total susceptible de haberlos recibido en ese mismo periodo.

El efecto de la regulación sucedida a partir del RD 661/2007 también es evidente observando las variables del Cuadro 3. Por un lado, el fuerte apoyo a la energía del RE se aprecia en términos de capacidad instalada, por encima de los $30 \mathrm{GW}$ desde el año 2009 y rozando los $40 \mathrm{GW}$ a cierre de 2013 , con casi $23 \mathrm{GW}$ de potencia eólica instalada. En segundo lugar, el hecho de disponer de mayores niveles de capacidad instalada, junto con la prioridad de despacho de este tipo de tecnologías, han

\section{CUADRO 3}

CAPACIDAD (GW), PRODUCCIÓN (TWh) E INCENTIVOS (€/MWh) DEL RÉGIMEN ESPECIAL

\begin{tabular}{|l|r|r|r|r|r|r|r|r|r|}
\hline \multirow{2}{*}{ Tecnología } & \multicolumn{3}{|c|}{$\mathbf{2 0 0 9}$} & \multicolumn{3}{c|}{$\mathbf{2 0 1 1}$} & \multicolumn{3}{c|}{$\mathbf{2 0 1 3}$} \\
\cline { 2 - 11 } & GW & TWh & $\begin{array}{c}\text { FIT/ } \\
\text { FIP }\end{array}$ & GW & TWh & $\begin{array}{c}\text { FIT/ } \\
\text { FIP }\end{array}$ & GW & TWh & $\begin{array}{c}\text { FIT/ } \\
\text { FIP }\end{array}$ \\
\hline Cogeneración & 5,91 & 22,02 & 47,58 & 6,16 & 25,04 & 57,27 & 5,97 & 25,35 & 71,76 \\
\hline Fotovoltaica & 3,65 & 6,20 & 424,63 & 4,24 & 7,39 & 324,35 & 4,64 & 8,28 & 309,63 \\
\hline Termosolar & - & - & - & 1,00 & 1,78 & 239,96 & 2,30 & 4,43 & 251,39 \\
\hline Viento & 18,84 & 38,23 & 42,35 & 21,02 & 41,67 & 40,98 & 22,79 & 54,48 & 43,98 \\
\hline Minihidroeléctrica & 2,01 & 5,44 & 42,99 & 2,03 & 5,26 & 39,00 & 2,04 & 7,03 & 43,06 \\
\hline Biomasa & 0,66 & 3,02 & 74,25 & 0,75 & 3,68 & 74,62 & 0,85 & 4,62 & 81,37 \\
\hline Residuos & 0,57 & 2,93 & 30,05 & 0,59 & 2,92 & 31,26 & 0,60 & 2,73 & 30,57 \\
\hline Tratamien. de residuos & 0,66 & 3,93 & 82,44 & 0,66 & 4,42 & 82,37 & 0,63 & 4,44 & 108,55 \\
\hline Total & 32,30 & 81,77 & 75,46 & 36,46 & 92,16 & 74,89 & 39,82 & 111,35 & 82,04 \\
\hline
\end{tabular}

NOTA: Datos de termosolar reportados a partir de 2010.

FUENTE: Elaboración propia a partir de datos de la CNE/CNMC (2002-2013) para 2009, 2011 y 2013. 
propiciado una intensificación considerable de la producción de energía de RE, con un incremento de un 36 por 100 entre 2009 y 2013, siendo especialmente destacado el aumento experimentado por tecnologías como el viento (un 42 por 100 entre 2009 y 2013) y la biomasa (un 53 por 100 para el mismo periodo). La cara negativa de este éxito de las renovables, en cambio, es su elevado coste en términos de primas. En 2013, cada megavatio de RE producido había costado al sistema 82,04 euros en media, cifra bastante superior en el caso de las tecnologías solares y algo más reducida en el caso de tecnologías eólica y mini-hidráulica. Este alto nivel de costes es el resultado combinado del aumento de producción de las tecnologías limpias y el sobredimensionamiento de los incentivos recibidos por algunas de ellas, donde las significativas variaciones existentes entre tecnologías y años se deben al distinto peso que cada una de ellas presenta en cada periodo, tanto en cuanto a producción como en nivel de incentivos (por ejemplo, en el caso de la cogeneración se observa el incremento de producción entre 2009 y 2013 con una subida de la prima unitaria). Adicionalmente, en el caso de la tecnología solar fotovoltaica se aprecia la reducción de horas de funcionamiento retribuidas aprobada en el RD 1614/2010, ya que en 2013 esta tecnología recibió un incentivo medio menor que en 2009, a pesar de haber incrementado su producción.

\subsection{Déficit de tarifa}

El déficit tarifario no es más que el resultado evidente de que los costes reconocidos del sistema eléctrico español son superiores a los ingresos obtenidos para el mismo periodo $^{\mathbf{1 0}}$. Su existencia, que no tiene paralelo en otras actividades económicas reguladas ${ }^{11}$, supone un lastre para el desarrollo normal de la actividad en el sector eléctrico. En su liquidación 14/2012 la CNMC cifraba en unos 5.609 millones de euros el déficit para 2012, cantidad que se situaba en 3.188 millones de euros para 2013, cifra aún elevada, pero sensiblemente inferior al de su año predecesor e incluso menor que la previsión inicial del gobierno para dicho ejercicio, cifrada en 3.500 millones de euros. El Gráfico 5 resume la evolución del déficit tarifario desde 2000.

\footnotetext{
${ }^{10}$ Hablamos de costes reconocidos, pero los costes reales de producción también han sufrido variaciones significativas en los últimos años. Por un lado, los costes del mercado diario han disminuido debido a la bajada de precios propiciada por la mayor participación de energía renovable en el sistema. Sin embargo, los costes de los servicios de ajuste de mercado han aumentado, dado el carácter intermitente de la mayor parte de la generación renovable. El balance global es que el precio de la electricidad fue menor en 2013 que en 2008 , por ejemplo, con una reducción de 12,6 euros por MWh según datos del operador del mercado.

${ }_{11} \mathrm{Si}$ bien es cierto que comienza a preocupar el déficit tarifario en el mercado de gas natural. De acuerdo con la liquidación 14 hecha por la CNMC para el año 2013, éste se elevaría a 326 millones de euros. Tanto este déficit, como los anteriores, y los que se pudieran generar posteriormente, se regulan mediante la Orden ITC/3993/2006, de 29 de diciembre.
} 


\section{GRÁFICO 5 \\ DÉFICIT DEL SISTEMA ELÉCTRICO GENERADO ENTRE 2000-2013 \\ (Millones de $€$ )}

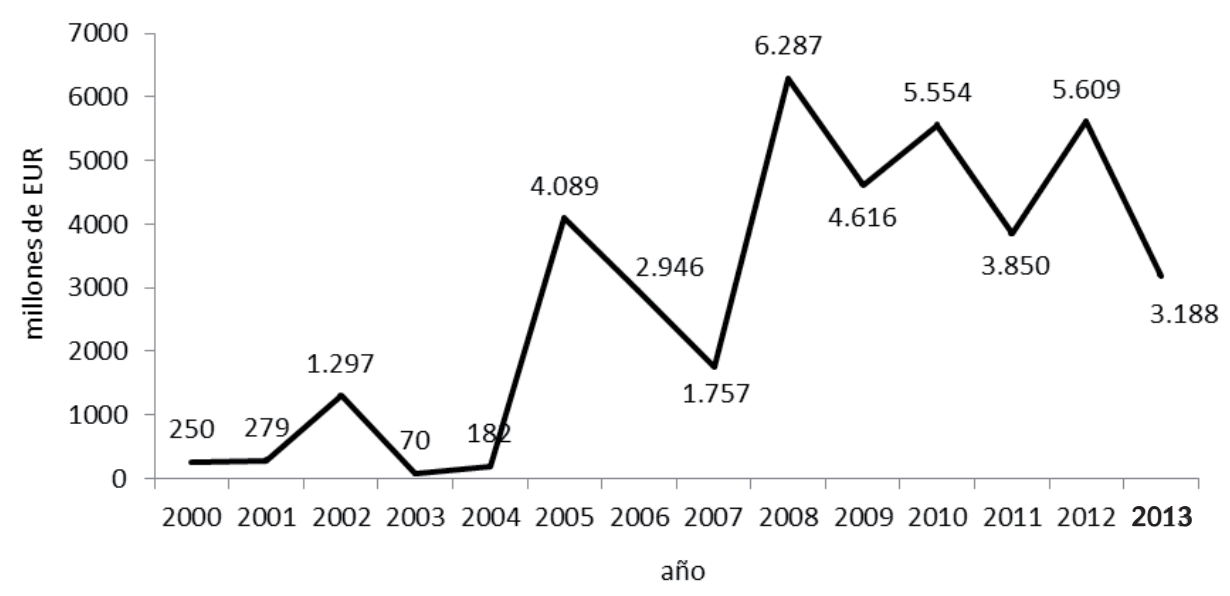

FUENTE: CNMC (2013).

El déficit es una deuda del sistema eléctrico con las empresas de generación que lo financian temporalmente ${ }^{12}$. Dicha deuda se traslada a generaciones de consumidores futuras a través del reconocimiento de derechos de cobro a largo plazo, y su cesión a terceros ha estado tradicionalmente permitida. Está cesión se realizó a través de titulizaciones durante el periodo 2000-2005. A partir de 2007 se decidió que la Comisión Nacional de Energía (CNE) gestionase la subasta de derechos de cobro, subasta que no tuvo buena acogida entre los mercados financieros, debido a las características de riesgo del producto. En 2009 se creó el Fondo de Amortización del Déficit Eléctrico (FADE) ${ }^{\mathbf{1 3}}$ con el fin de financiar y amortizar el importe acumulado de la deuda del sistema público de liquidaciones eléctrico con las compañías generadoras. El funcionamiento de este fondo es el siguiente. Una vez han sido cedidos los derechos de cobro por parte de los tenedores del déficit al FADE, éste los transforma en distintos valores de renta fija susceptibles de negociarse en los mercados de valores. Todos los derechos sirven de respaldo para el pago de las obligaciones derivadas de los bonos emitidos, por lo que el FADE no sólo sirve para titulizar nueva deuda, sino que también se puede utilizar para refinanciar series anteriores (para más información sobre el FADE y su funcionamiento ver de los Llanos Matea, 2013).

${ }^{12}$ Hasta la aprobación de la Ley 24/2013 los desfases eran financiados por las cinco grandes compañías del sistema eléctrico. Posteriormente serán financiados por todos los sujetos del sistema de liquidación en función de los derechos de cobro que generen.

13 Ver RD-1 6/2009. 
Según datos de la propia CNMC, la deuda viva a 8 de noviembre de 2013 se situaba en 25.456,89 millones de euros, de los cuales el 88,51 por 100 pertenecían al FADE y el 11,49 por 100 a terceros. En ese momento la deuda quedaba completamente saldada con las compañías eléctricas e incrementada con el FADE (CNMC, 2013).

Uno de los objetivos fundamentales de las sucesivas reformas energéticas aprobadas por el gobierno busca eliminar el déficit de actividades reguladas, a partir de la reducción de los costes de acceso del sistema eléctrico ${ }^{14}$. Estos costes regulados se desglosan a su vez en dos tipos: (i) los costes inherentes al sistema, como transporte y distribución; y (ii) otros costes asociados, entre los que se incluyen las primas al ya extinto RE y el pago de anualidades del déficit tarifario, entre otros. Según muestra el Gráfico 6, los costes de acceso en España han ido creciendo considerablemente desde el año 2000, crecimiento que se acentúa especialmente a partir del año 2008, debido fundamentalmente al aumento de la cuantía dedicada al apoyo del RE y a las anualidades de déficits acumulados.

\section{GRÁFICO 6}

\section{EVOLUCIÓN DE LOS COSTES DE ACCESO (MILLONES DE €)}

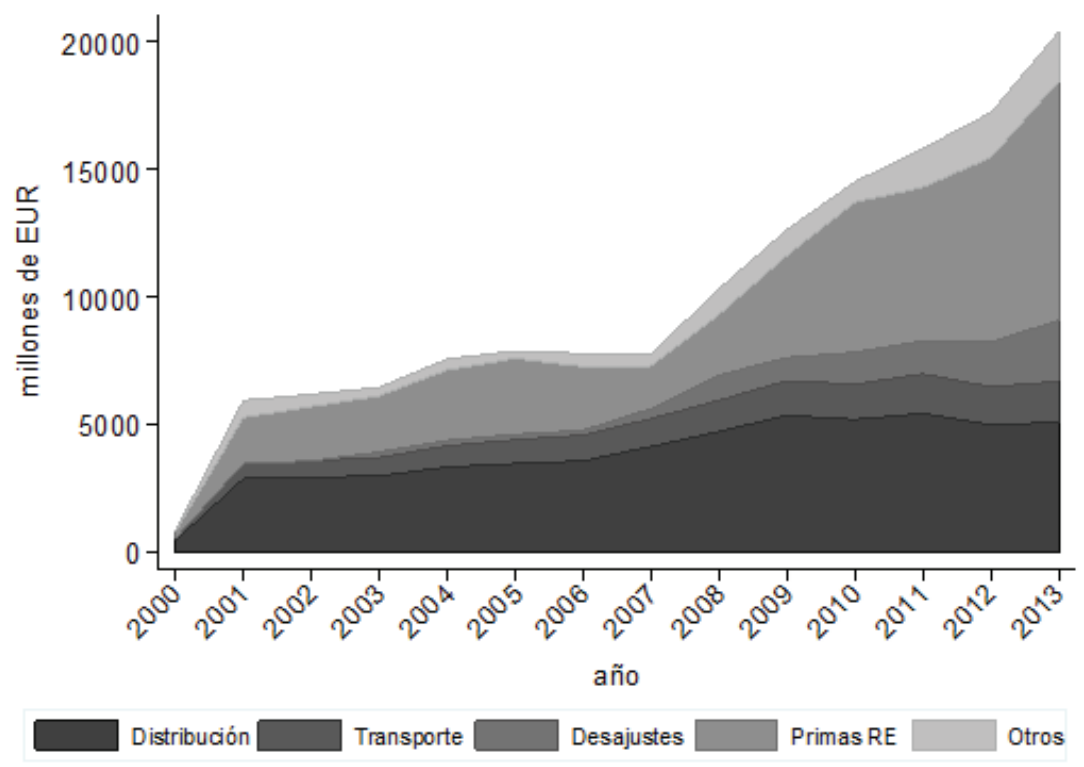

FUENTE: Elaboración propia a partir de las liquidaciones definitivas publicadas por la CNE durante los años 2000-2007 (CNE, 2000-2007) y la Liquidación n ${ }^{\circ} 14$ publicada por la CNMC durante el periodo 2008-2013 (CNE/ CNMC, 2008-2013).

14 Los costes de acceso o peajes son parte de los costes regulados del sistema eléctrico, a los que también se suman el alquiler de equipos de medida y control, así como los impuestos. 
Hasta el año 2007 el incremento del déficit se debía a la diferencia entre el precio fijado para la energía en la tarifa y su coste real en mercado. Este desajuste pudo ser corregido con las subastas CESUR (ver sección 1.1) y desde 2008 la principal causa del incremento del déficit es la insuficiencia de la parte regulada de la tarifa para cubrir los costes reconocidos. En este sentido, cabe destacar que este aumento exponencial del déficit tarifario coincide con la implementación del RD 661/2007, en el que se establecieron nuevos niveles de subsidios (mayores para biomasa y cogeneración), así como un suelo y un techo para la remuneración del RE. Este decreto supuso el verdadero salto en la promoción de instalaciones de energía renovable y cogeneración (pasando de 24,8 GW de RE instalados en 2007 a 39,8 GW en 2013 (CNE/CNMC, 2002-2013)), lo que conllevó un fuerte incremento de producción de RE (de 58,2 TWh vendidos en 2007 a 111,4 TWh en 2013 (CNE/CNMC, 20022013)), y se tradujo finalmente en un elevado gasto en incentivo ${ }^{15}$ que ha motivado, en parte y con objeto de contención de gasto, la reforma del RD 413/2014.

A pesar de las sucesivas reformas del sistema eléctrico en España, las tarifas que pagan los consumidores finales a día de hoy, a diferencia de lo que ocurre en otros países ${ }^{16}$, siguen sin incorporar la totalidad de los costes del sistema eléctrico, situación agravada por el importante descenso de la demanda causado por la crisis económica acaecida desde 2010.

\section{Nuevo marco regulatorio}

El resultado de la 25 subasta CESUR junto con el crecimiento desbocado del déficit de tarifa, fueron los dos detonantes que finalmente impulsaron la reforma del sistema. El nuevo marco regulatorio comenzó a gestarse en diciembre de 2013, con la aprobación de la nueva Ley del Sector Eléctrico 24/2013 que reformaba la Ley 54/1997 en varios aspectos. Desde el punto de vista de la competencia, la ley introduce cambios tanto en el mercado mayorista como en el mercado minorista de electricidad. En particular, esta ley propone medidas para garantizar la sostenibilidad económica y financiera del sector eléctrico, buscando (i) el equilibrio entre costes e ingresos (eliminar el déficit tarifario), (ii) establecer con claridad la financiación de los costes, (iii) definir un nuevo régimen retributivo del sistema eléctrico, (iv) la regulación de las relaciones entre los consumidores y las empresas comercializadoras (precios al pequeño consumidor, tarifas de último recurso...) y (v) otros asuntos como la garantía de suministro eléctrico, el autoconsumo (pendiente de regulación a día de hoy), la regulación de las actividades en territorios no peninsulares y la integración de los mercados europeos (participación en la iniciativa Price Coupling

15 A pesar del coste del sistema de retribución, la participación del RE en el mercado eléctrico ha traído consigo otra serie de ventajas en cuanto a reducción de los precios del pool. Para más información sobre el tema ver CIARRETA et al. (2014).

${ }^{16}$ En Alemania, por ejemplo, el coste de las energías renovables se repercute directamente al consumidor final y se considera como una inversión de la sociedad para el desarrollo de las tecnologías limpias. 
of Regions). En los siguientes apartados nos enfocamos en los tres puntos más destacados de la reforma: la tarificación para consumidores finales, las energías renovables y la gestión del déficit tarifario.

\subsection{La nueva tarificación eléctrica para consumidores finales}

El Real Decreto 216/2014, de 28 de marzo, regula los comercializadores de referencia y establece la metodología de cálculo de los Precios Voluntarios para el Pequeño Consumidor (PVPC) de energía eléctrica y su régimen jurídico de contratación. El PVPC serán los precios máximos que podrán cobrar los comercializadores de referencia a los consumidores que se acojan a dicho precio (ver artículo 17 de la Ley 24/2013). Salvo manifestación expresa en contra por parte del consumidor, la modalidad de contratación con el comercializador de referencia será a PVPC. El nuevo sistema entró en vigor desde el 1 de abril de 2014.

La anomalía existente entre la fijación del precio para el consumidor final y el precio del mercado mayorista se subsana en parte con la reforma. Así el artículo 10 del RD 216/2014 establece que el precio medio horario $\left(P_{h}\right)$ se calcula como media ponderada por las cantidades entre el precio del mercado diario, $P_{h}^{D}$, y el resultante de las diferentes sesiones del mercado intradiario, $P_{i}^{h}{ }^{17}$.

$$
P_{h}=\frac{P_{h}^{D} Q_{h}^{D}+\sum_{i \in I} P_{h}^{i} Q_{h}^{i}}{Q_{h}^{D}+\sum_{i \in I} Q_{h}^{i}}
$$

Por lo tanto, el coste final de adquisición de la electricidad para el período de facturación $p$ que incluye $H$ horas sería la suma de la parte correspondiente al mercado al contado, esto es del precio fijado en el mercado mayorista junto con los precios de las correspondientes sesiones del mercado intradiario, el coste de los servicios de ajuste y pagos por capacidad, además de los peajes fijados periódicamente de manera administrativa. Así pues el coste de producción se determina como media ponderada del precio medio horario, $P_{h}$, coste de los servicios de ajuste, $S A_{p, h}$, y los coeficientes de pérdida, $P E R D_{p, h}$.

$$
C F_{p}=\frac{\sum_{h=1}^{H}\left(\left(P_{h}+S A_{p, h}\right)\left(1+P E R D_{p, h}\right)\right) Q_{p, h}}{\sum_{h=1}^{H} Q_{p, h}}
$$

Aun siendo pronto para evaluar el impacto de las medidas implementadas, podemos ver la evolución de los precios mayoristas de la energía en relación con sus homólogos europeos. Si el precio reflejara el coste marginal de generación y dado que

${ }^{17}$ Desde el punto de vista empírico para el cálculo del PVPC no existe diferencia significativa entre utilizar únicamente los precios y cantidaddes de equilibrio de la primera sesión del mercado intradiario o los resultantes de las seis sesiones. 
éste es similar en los diferentes mercados europeos considerando áreas e intervalos horarios equivalentes, deberíamos observar un patrón similar de comportamiento. Sin embargo, la existencia de importantes restricciones en las interconexiones hace que las diferencias en los precios sean aún significativas y la convergencia en precios limitada ${ }^{18}$.

La CNMC en su informe del 25 de febrero de 2014 (CNMC, 2014) sobre el RD ya señala algunas de las ventajas y de las desventajas del nuevo sistema. Entre las ventajas, en primer lugar la prima de riesgo de los mercados a plazo no se transfiere a los consumidores finales tal y como sucedía con las subastas trimestrales. En segundo lugar, el mecanismo de fijación de precios transmite a los consumidores finales una señal mucho menos distorsionada del valor de la electricidad de lo que eran anteriormente. En tercer lugar, se posibilita la implementación de mecanismos de gestión de la demanda mucho más eficientes que los existentes. Entre las desventajas, destaca el hecho de que a pesar de que los precios son conocidos ex ante por los consumidores, de tal forma que puedan anticipar sus decisiones de consumo, no resultan fácilmente accesibles a todos ellos. Es más, resulta difícil para un consumidor final acceder a los distintos productos ofrecidos por las comercializadores y en su caso cambiar de proveedor. En esta línea resulta interesante la iniciativa tanto de la CNMC como de OMIE para publicitar precios, aunque no creemos que los pequeños consumidores accedan a ellos con facilidad. En segundo lugar, la variabilidad en los precios para el consumidor final es mayor con respecto al precio trimestral constante del sistema anterior lo cual distorsiona la toma de decisiones de consumo óptimas.

\subsection{El nuevo sistema de retribución a la energía renovable y la cogeneración}

El Real Decreto 413/2014, de 6 de junio, regula el nuevo régimen jurídico y económico de la actividad de producción eléctrica mediante energías renovables, cogeneración y residuos ${ }^{19}$, al que se suma la Orden IET/1045/2014, de 16 de junio, en la que se aprueban los parámetros retributivos de las instalaciones tipo aplicables. De esta manera se conforma el nuevo marco regulatorio para las fuentes renovables, cogeneración y residuos, ya iniciado con el RD-1 9/2013 y la Ley 24/2013, quedando derogando definitivamente el régimen de retribución establecido en la Ley 54/1997 y el RD 661/2007.

${ }^{18}$ Esta es una de las principales conclusiones en CIARRETA y ZÁRRAGA (2014). Utilizando un modelo multivariante de transmisiones de volatilidad entre el EPEX, mercado diario que agrupa a Francia, Alemania, Suiza y Austria, y OMIE muestran como la falta de interconexiones es un elemento fundamenteal para explicar la no convergencia de precios.

${ }^{19}$ Los términos Régimen Ordinario y RE han sido eliminados en la Ley 24/2013, de 26 de diciembre, pero se mantiene un régimen retributivo específico de las actividades de transporte, distribución y producción a partir de energías renovables, cogeneración y residuos, para que estas tecnologías puedan competir en igualdad con el resto de instalaciones en el mercado. 
El principal objetivo del nuevo marco regulatorio es garantizar la estabilidad del sistema eléctrico, basado en el principio de rentabilidad razonable sobre una serie de instalaciones tipo, para que las tecnologías renovables y de cogeneración cubran los costes que no pueden recuperar en el mercado sin incurrir en una carga excesiva para el sistema. La retribución de cada instalación será, por tanto, la resultante de la aplicación de las siguientes fórmulas durante el tiempo de vida útil regulatoria de la instalación tipo ${ }^{20}$ :

$$
\begin{gathered}
R_{i}=R V_{p m}+R_{e}+\left[l_{i n v}\right] \\
R_{e}=R_{i n v}+R_{o} \\
R_{i n v}=C l_{i t} \\
R_{o}=C E_{i t}-I M_{i t}
\end{gathered}
$$

Donde $R_{i}$ indica retribución de la instalación, $R V_{p m}$ es la retribución de venta a precio de mercado, $R_{e}$ se refiere a una retribución específica e $I_{i n v}$ es el incentivo a la inversión para instalaciones en sistemas eléctricos no peninsulares que supongan reducción global del coste de generación. La $R_{e}$, a su vez, depende de la retribución a la inversión $\left(R_{i n v}\right)$, que se calcula a partir de los costes de inversión de instalación tipo no recuperados $\left(C I_{i}\right)$, y de la retribución a la operación $\left(R_{o}\right)$, obtenida como la diferencia entre los costes de explotación de la instalación tipo $\left(C E_{i t}\right)$ y los ingresos de mercado de la instalación tipo $\left(I M_{i}\right)$.

Adicionalmente, serán parámetros retributivos todos aquellos empleados para el cálculo de los anteriores, siendo los más destacados:

a) Valor estándar de la inversión inicial de la instalación tipo.

b) Estimación del precio de mercado diario e intradiario.

c) Número de horas de funcionamiento de la instalación tipo.

d) Estimación del ingreso futuro por la participación en el mercado de producción.

e) Otros ingresos de explotación definidos en el artículo 24 (por ejemplo, posibles convocatorias de ayudas públicas, cánones de eliminación de residuos, ingresos procedentes de la producción de calor útil asociado...).

f) Estimación del coste futuro de explotación.

g) Tasa de actualización que toma como valor el de la rentabilidad razonable.

h) Coeficiente de ajuste de la instalación tipo.

i) Valor neto del activo.

${ }^{20}$ A cada instalación tipo le corresponderá un conjunto de parámetros retributivos que se calcularán por referencia a la actividad realizada por una «empresa eficiente y bien gestionada», que concretarán el régimen retributivo específico y permitirán la aplicación del mismo a las instalaciones asociadas a dicha instalación tipo. La orden propone, entre otros, 806 tipologías de instalaciones de cogeneración, 576 de fotovoltaicas y 23 de eólicas. 
El concepto de rentabilidad razonable de proyecto se establece en el entorno del rendimiento medio de las Obligaciones del Estado a diez años (incrementado con un diferencial) de los 24 meses previos al mes de mayo del año anterior al de inicio del periodo regulatorio. Para las instalaciones ya existentes, el diferencial será de 300 puntos básicos sobre el rendimiento medio en el mercado secundario de los diez años anteriores a la entrada en vigor del RD 413/2014.

En este decreto se establecen periodos regulatorios de seis años de duración, que a su vez se dividen en dos semiperiodos regulatorios de tres años. El primer periodo regulatorio se refiere al comprendido entre la fecha de entrada en vigor del RD-1 9/2013 y el 31 de diciembre de 2019, mientras que el primer semiperiodo regulatorio se corresponde con el existente entre la fecha de entrada en vigor del RD-1 9/2013 y el 31 de diciembre de 2016.

Los parámetros retributivos podrán ser revisados al finalizar cada semiperiodo o periodo regulatorio. En la revisión que corresponda a cada período regulatorio se podrán modificar prácticamente todos los parámetros retributivos, incluyendo el valor sobre el que girará la rentabilidad razonable en lo que reste de vida regulatoria de las instalaciones tipo. Los únicos parámetros que no se permite modificar, una vez definidos, son la vida útil regulatoria y el valor estándar de la inversión inicial de una instalación tipo. Adicionalmente, anualmente se actualizarán los valores de retribución a la operación para aquellas tecnologías cuyos costes de explotación dependan esencialmente del precio del combustible.

En las revisiones de los semiperiodos regulatorios se revisarán para el resto del periodo regulatorio, las estimaciones de precios de mercado de producción (ajustados a precios reales de mercado) y los ingresos por la venta de la energía generada (valorada al precio del mercado de producción), en función de la evolución de los precios del mercado y las previsiones de horas de funcionamiento de la instalación. En el cálculo de los parámetros retributivos se definen también unos límites superiores e inferiores para la estimación de venta de energía, término denominado valor de ajuste por desviaciones en el precio del mercado, que se compensará a lo largo de la vida útil de la instalación y que generará un saldo positivo o negativo cuando el precio medio anual del mercado diario e intradiario se sitúe fuera de dichos límites.

El número de horas de funcionamiento de un periodo concreto vendrá determinado por el cociente entre la energía vendida en mercado y la potencia instalada. Si durante un año la instalación presenta un número de horas equivalentes de funcionamiento inferior al umbral de su instalación tipo, podría llegar a perder el derecho al régimen retributivo específico.

Por último, en cuanto a la vida útil de las instalaciones tipo, se consideran 20 años para cogeneración y eólica, 25 años para termo-solar, mini-hidroeléctrica, biomasa y biogás y 30 años para la tecnología solar fotovoltaica. Una vez que las instalaciones superen la vida útil regulatoria, podrán mantenerse en operación, pero dejarán de percibir la retribución a la inversión y la retribución a la operación, ingresando exclusivamente retribución obtenida por la venta de energía en el mercado. 


\subsection{Evolución reciente de la normativa sobre déficit tarifario}

En diciembre de 2012 comenzaron a adoptarse nuevas medidas fiscales sobre la producción de energía para compensar el déficit con la aprobación de la Ley $15 / 2012$, en la que se instauraba un impuesto sobre el valor de la producción eléctrica. Posteriormente, en febrero de 2013 (RD-1 2/2013) el Gobierno propuso una simplificación del sistema de apoyo a las energías renovables y revisó el ajuste anual de los precios regulados, de nuevo con intención de disminuir el déficit. Finalmente, y como preludio de la Ley 24/2013, el RD-1 9/2013 incluyó un nuevo paquete de medidas reductoras del déficit, entre las que se encontraban (i) la sustitución del régimen de primas a las renovables por un sistema de retribución aplicable con carácter retroactivo y basado en la rentabilidad razonable de la inversión de una «empresa eficiente y bien gestionada», (ii) la modificación de la retribución de las actividades de distribución y transporte, (iii) la introducción de nuevos criterios de distribución del bono social y (iv) la autorización de la revisión de los ingresos en caso de incremento de costes.

Finalmente, la Ley 24/2013 del Sector Eléctrico continúa con la intención de control del déficit tarifario. Algunos de los aspectos destacados de esta ley es que reduce gran parte de la financiación pública ${ }^{21}$ dedicada al déficit, y limita los desajustes por déficit de ingresos de un ejercicio al 2 por 100 de los ingresos estimados para dicho periodo, y la deuda acumulada por desajustes de años anteriores al 5 por 100 de los ingresos estimados para ese ejercicio. En este sentido, los desajustes que se produzcan (por debajo de los umbrales antes mencionados) entre costes e ingresos serán corregidos mediante revisiones automáticas de los peajes y cargos, y lo no compensado será financiado por todos los sujetos del sistema de liquidación, cada uno en proporción sus derechos de cobro. El déficit futuro, por tanto, no quedará exclusivamente en manos de los cinco grandes operadores, como ocurría hasta ahora ${ }^{22}$. Además, a partir de 2013 se cancela la posibilidad de cesión de tales derechos (por déficit de ingresos) al Fondo de Titulización del Déficit del sistema.

\section{Efectos de la nueva regulación}

\subsection{Efectos sobre el precio de los consumidores}

La eficiencia en la asignación de recursos se obtiene si el mecanismo de precios transmite las señales adecuadas de escasez. Estos precios deben de estar ligados a los costes de producción. Es éste precisamente uno de los objetivos de la reforma ya que, tal y como se puede ver en los Gráficos 1 y 2, el organismo regulatorio argüía que había margen para que el precio medio pudiera descender.

\footnotetext{
${ }^{21}$ Se suprime el crédito extraordinario de 2.200 millones de euros previsto en la Ley 15/2013 y se suprime la financiación pública destinada al coste extra asociado a los territorios no peninsulares.

${ }^{22}$ El déficit generado durante 2013 aún seguirá siendo financiado por las cinco grandes empresas del sector eléctrico nacional.
} 
GRÁFICO 7

EVOLUCIÓN PRECIO MEDIO DIARIO MESES JULIO-SEPTIEMBRE 2012, 2013, 2014

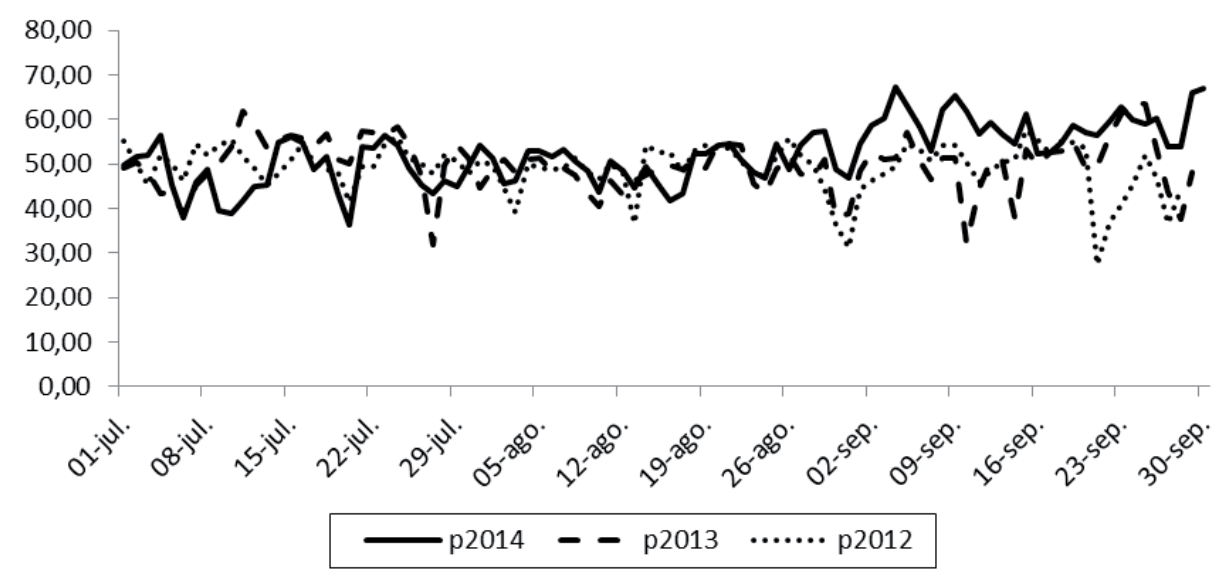

NOTAS: Precios medios aritméticos diarios (PDBC). Hacemos corresponder el día de la semana de cada año, p.ej. martes 1 julio.

FUENTE: Elaboración propia a partir de datos de OMIE.

Aún resulta pronto para poder comparar el efecto de la reforma en la evolución de los precios finales, pero podemos observar ya los primeros resultados. En el Gráfico 7 se representa la evolución diaria del $P_{h}^{D}$ entre los meses de julio y septiembre de 2012, 2013 y $2014^{23}$. Se observa como el nivel medio de los precios entre julio y septiembre es en media superior en 2014 con respecto a 2012 y 2013. Por lo tanto, de momento la reforma ha provocado un alza de precios en comparación con el período CESUR, lo cual iría en contra de los argumentos para realizar la reforma. Un análisis más exhaustivo requeriría filtrar los componentes predecibles y estacionales del proceso de precios de tal forma que quede el componente impredecible o estocástico que es el que se debería utilizar como medida adecuada de la volatilidad de los precios. Con estos datos podríamos analizar la posible existencia de prácticas anticompetitivas.

La evolución a partir del 1 de abril de 2014 de los precios en el mercado diario y en el mercado intradiario presenta sin embargo una tendencia creciente. Así el PVPC calculado para el período julio a septiembre de 2014 es de 52,36 euros por MWh, un

${ }^{23}$ También hemos representado $P_{h}$ y no hay diferencias significativas entre las diferencias de ambos precios. El precio $P_{h}$ para antes de 2014 sería un precio calculado utilizando como supuesto de comportamiento de los agentes la existencia de dichas subastas; por lo tanto, no se puede concluir que en ausencia de las mismas ese precio hubiese sido el de equilibrio. Por este motivo reportamos los precios efectivos del PDBC. 
5 por 100 más alto que en 2013 para el mismo período ${ }^{24}$. Pero tomando únicamente el mes de septiembre, el precio es 17,51 por 100 más elevado que el mismo mes del año anterior. Por lo tanto, aun siendo pronto para establecer una valoración, el objetivo de reducir precios no se está alcanzando.

\subsection{Efectos sobre la producción de energía renovable}

La supresión de las primas y tarifas ha creado un clima de inseguridad jurídica que desalienta la inversión en el sector renovable, dificultando el cumplimiento de los objetivos energéticos y climáticos en el marco de la Estrategia Europa 2020, y aumentando la dependencia de energía importada (situada en un 79 por 100 en 2012, valor superior a la media europea del 54 por 100, CE (2012)).

A nivel de mercado, algunos efectos del RD 413/2014 incluyen posibles subidas de precios debidas al incremento de las pujas de las instalaciones de energía renovable y cogeneración, una vez eliminada la limitación de oferta de energía a precio cero, y viendo que en ausencia de incentivos algunas plantas podrían no compensar sus costes de producción con los precios actuales (sobre todo aquellas que requieran el uso de combustible, como son las plantas de cogeneración). Adicionalmente, la participación de las fuentes de energía renovable en los mercados intradiarios podría verse aumentada, afectando negativamente a los costes del sistema. Por otro lado, cabe destacar también una posible reducción de la capacidad renovable con el inminente cierre de algunas instalaciones, principalmente solares, que con la nueva regulación no van a poder hacer frente a sus compromisos financieros.

Aún es pronto para tener datos empíricos al respecto (la reforma se instauró en junio de 2014 y en el momento de preparación del presente documento sólo hay datos publicados hasta mediados de octubre), pero lo que sí se puede concluir a día de hoy es que el precio medio en septiembre de 2014 ha sido superior al de los dos ejercicios anteriores (ver Gráfico 7), aunque aún no es posible determinar si el efecto se debe al nuevo sistema de precios a consumidores finales, al cambio de sistema de incentivos a energía renovable y cogeneración o a otros posibles efectos.

El Gráfico 8 representa la evolución de la producción de energía renovable y cogeneración del tercer trimestre (julio, agosto, septiembre) en el programa diario base de funcionamiento, que incluye la energía negociada en el mercado diario más los contratos bilaterales con entrega física de energía.

${ }^{24}$ Durante el período de existencia del MIBEL, solamente en 2008 el precio medio fue superior, donde se llegó a los 70,43 euros por MWh. 
GRÁFICO 8

EVOLUCIÓN DE LA PRODUCCIÓN DE ENERGÍA RENOVABLE Y COGENERACIÓN (GWh)

Julio

॥ $2012 \square 2014$

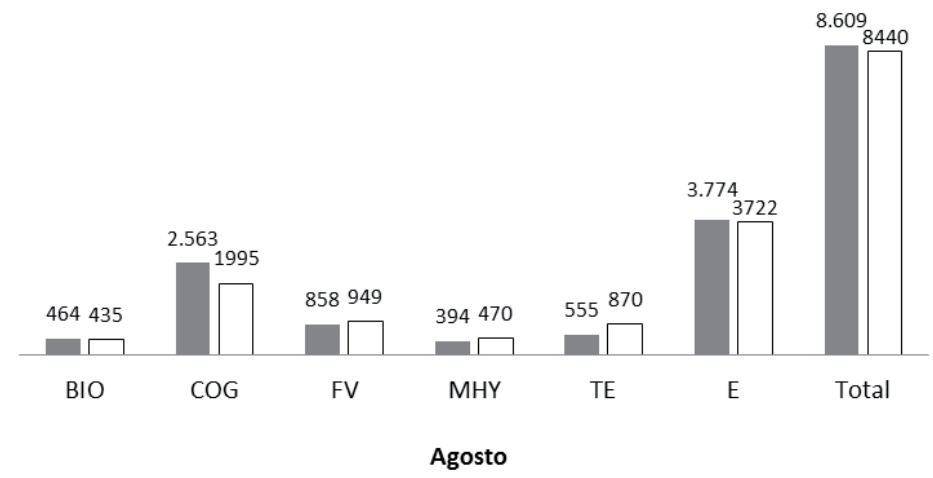

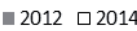

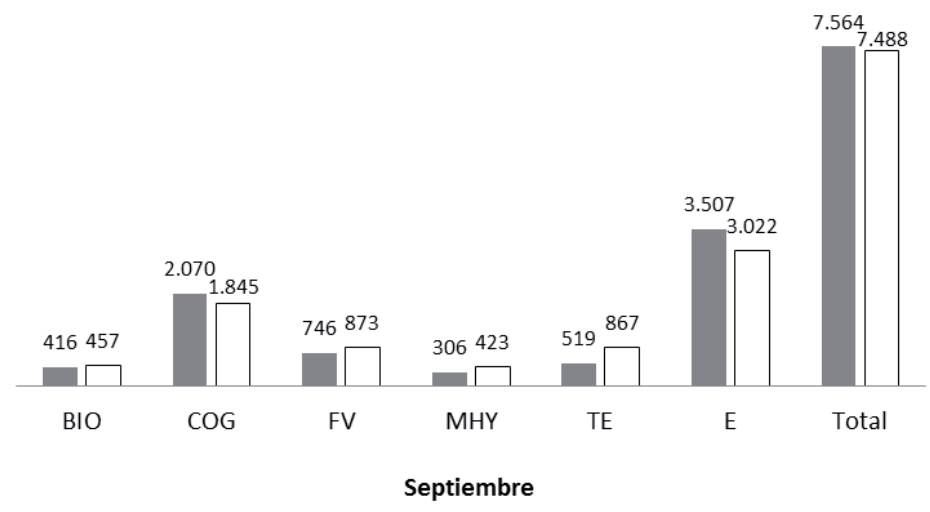

— $2012 \square 2014$

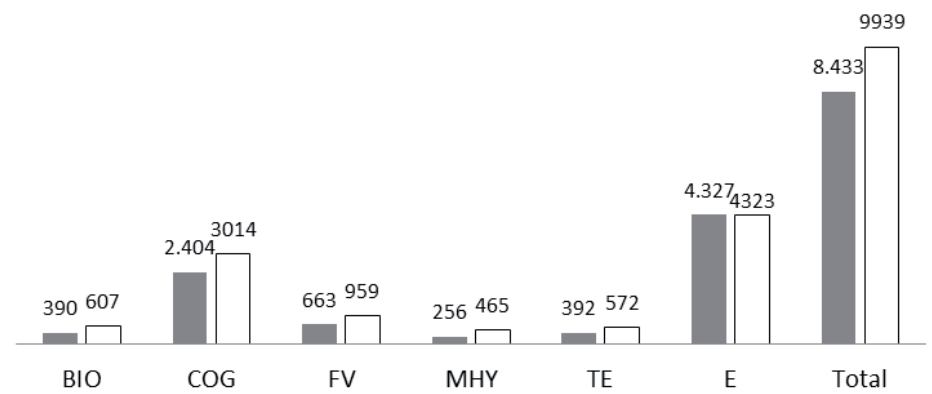

FUENTE: Elaboración propia a partir de datos de REE. Incluye producción del programa diario base de funcionamiento (mercado diario + contratos bilaterales con entrega física). BIO=Biomasa, COG=Cogeneración, $\mathrm{FV}=$ Solar fotovoltaica, $\mathrm{MHY}=$ Mini-hidroeléctrica, TE=Termo-solar, E=Eólica. 
Vista la evolución para las distintas tecnologías se puede concluir que para los meses de julio y agosto no ha habido diferencias sustanciales en la producción de fuentes renovables y cogeneración entre los años 2012 y 2014, teniendo en cuenta que en 2012 aún estaba en vigor el sistema de primas y tarifas, mientras que en julio de 2014 ya se aplicaba la nueva retribución. Para septiembre, en cambio, aparece reflejado un incremento en la producción total de energía renovable y cogeneración que, aunque es cierto que podría deberse a causas externas como la meteorología, la demanda o la propia estacionalidad del año, también podría indicar que las generadoras están comenzando a cambiar su comportamiento en el mercado mayorista, promoviendo un alza de los precios.

Finalmente, según la Comisión Europea (CE, 2012), la reducción del coste de la energía renovable podría hallarse más allá de la energía renovable, resaltando conceptos como la simplificación de los complejos procedimientos de autorización y planificación, cuestión aún pendiente para España. Adicionalmente, el bajo nivel de interconexión con sus países vecinos (Francia y Portugal) resulta perjudicial para la competencia del sector. Un aumento de capacidad de intercambios transfronterizos permitiría equilibrar la oferta de energías renovables (sobre todo eólica), al reducir los costes asociados a energías de reserva y transmisión de electricidad.

\subsection{Efectos sobre el déficit tarifario}

Para apreciar un posible efecto de las últimas medidas tomadas en la reforma del sistema eléctrico representamos en el Cuadro 4 la comparativa de las últimas cuatro liquidaciones número 7, ya que es la última disponible para 2014 en la fecha de realización del presente documento. Antes de realizar ninguna comparativa al respecto, es necesario destacar que la liquidación número 7 (la séptima liquidación del año) abarca los meses comprendidos entre enero y julio y, por tanto, puede sufrir modificaciones con respecto a la última liquidación del año, debido a que los ajustes de las distintas partidas pueden variar a lo largo de las siguientes liquidaciones dada la diferencia de estacionalidad entre ingresos y costes del sistema. De hecho, observando el periodo 2010-2013 dentro del Cuadro 4 se aprecia que para algunos años (2010-2012) el déficit final ha sido superior al de la liquidación 7, mientras que para otros (2013) ha sido inferior. 


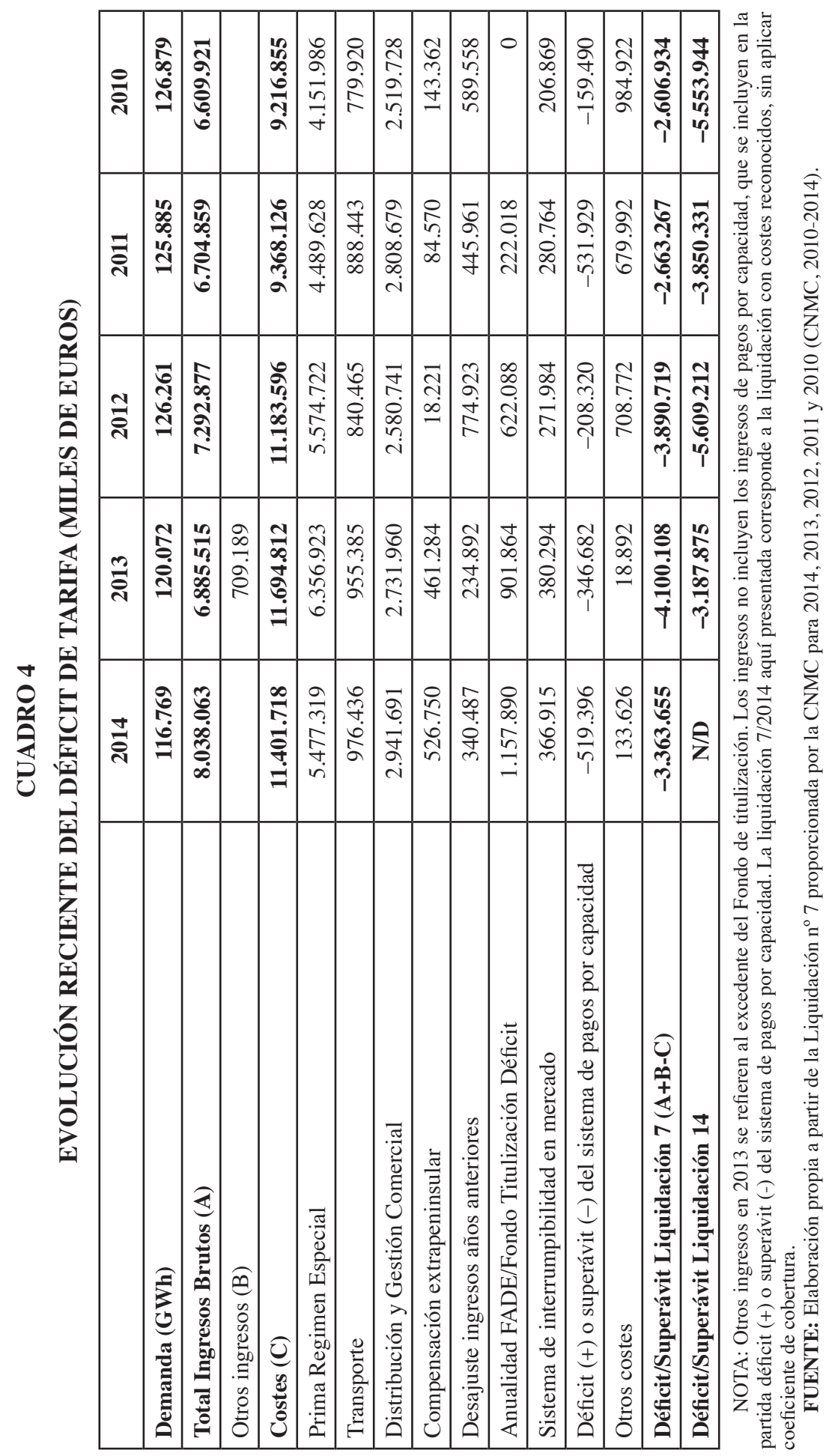


Vistos los resultados de esta liquidación provisional, se puede apreciar que las nuevas medidas regulatorias están teniendo resultados, en cuanto al aumento de la partida correspondiente a ingresos del sistema, que en 2014 es superior a la de la liquidación equivalente de los años anteriores, a pesar de la considerable caída de la demanda. Además, el sistema se ha beneficiado de un menor coste de los pagos por capacidad. En cambio, no se detectan efectos en cuanto a reducción de costes: los costes del $\mathrm{RE}^{25}$ siguen siendo altos e incluso han aumentado los gastos asociados a distribución y gestión comercial con respecto a otros años. El dato relativo al RE no es totalmente indicativo, ya que las reliquidaciones negativas ${ }^{26}$ que resultan de la aplicación de octava disposición transitoria del RD 413/2014 no tendrán impacto hasta la liquidación número 8 de 2014. En este sentido, la cantidad total que la CNMC estima se deberá abonar por este concepto asciende a más de 1.236 millones de euros, habiéndose facturado en la Liquidación 7/2014 alrededor de 72 millones, por lo que en las siguientes liquidaciones este coste asignado irá disminuyendo.

Según estos resultados se podría decir que la nueva regulación no está teniendo aún efectos muy destacados en el déficit de tarifa. En cualquier caso, para poder extraer conclusiones definitivas habrá que esperar a la liquidación final de año, ya que quedan aún muchos ajustes pendientes de realizarse.

\section{Conclusiones}

El panorama del sector eléctrico español se encuentra ante intensos cambios regulatorios con la prioridad de mejorar la eficiencia y eliminar el déficit tarifario. La nueva regulación busca incrementar la eficiencia del sistema en un contexto de necesaria austeridad presupuestaria.

El año 2013 terminó con un déficit tarifario rondando los 3.000 millones de euros y una última subasta CESUR para el suministro de electricidad del primer trimestre de 2014 sospechosa de manipulación, que finalmente fue cancelada. Así, la aprobación en diciembre de 2013 de la nueva Ley del Sector Eléctrico ha llevado consigo cambios significativos en las políticas de demanda de consumidores finales, el apoyo a las energías renovables y la gestión del déficit tarifario.

El nuevo sistema de tarificación busca que los precios finales de la electricidad reflejen realmente los costes de producción basados en el orden de mérito de las distintas tecnologías. Mientras que los cambios en el apoyo a la generación mediante fuentes renovables se basan en la madurez de algunas tecnologías que están en

${ }^{25} \mathrm{Si}$ consideramos el coeficiente de cobertura, el coste del RE asciende aproximadamente a 3.757 millones de euros, menor que el presentado en el Cuadro 5. El coeficiente de cobertura en la liquidación n. ${ }^{\circ} 7$ de 2014 es del 68,59 por 100 y se debe a los desajustes temporales entre ingresos y costes del sistema contemplados en el artículo 19 de la Ley 24/2013.

${ }^{26}$ Es decir, lo que tendrán que devolver los productores del antiguo RE por las primas liquidadas en el periodo de transición entre decretos. 
condiciones de competir con las no-renovables y en la racionalización del apoyo a las tecnologías menos maduras.

Si bien es cierto que el espíritu de la reforma va en la línea de crear un mercado competitivo, y aunque aún es pronto para evaluar su efectividad, ciertos indicadores clave señalan que todavía peligran los objetivos marcados y que medidas complementarias serían necesarias para lograr un mercado realmente competitivo. Entre otras, mayor apoyo a las interconexiones entre España y sus países vecinos, con objeto de aumentar la eficiencia del sistema al reducir costes asociados a la energía de reserva y a la transmisión de electricidad; evitar la retroactividad en las medidas regulatorias, ya que genera un clima de desconfianza que se traslada al funcionamiento de todo el sector; o plantear una reforma estructural que regule entre otros la generación distribuida. Mientras sigan sin corregirse, no ganaremos en eficiencia y el problema del déficit de tarifa persistirá. El reto precisamente radica en conseguir un compromiso entre la contención del gasto y el diseño de unas tarifas que reflejen los costes reales.

\section{Referencias bibliográficas}

[1] CIARRETA, A.; ESPINOSA, M.P. y PIZARRO-IRIZAR, C. (2014): «Is green energy expensive? Empirical evidence from the Spanish electricity market», Energy Policy, 69, pp. 205-215.

[2] CIARRETA, A. y ZARRAGA, A. (2014): «Analysis of Mean and Volatility Price Transmissions in the MIBEL and EPEX Electricity Spot Markets», Energy Journal (forthcoming).

[3] CE(2012): COMISIÓN EUROPEA: Documento de trabajo de los servicios de la comisión de evaluación del programa nacional de reforma y del programa de estabilidad de España para 2012 que acompaña al documento Recomendación de RECOMENDACIÓN DEL CONSEJO relativa al programa nacional de reforma de 2012 de España y por la que se emite un dictamen del Consejo sobre el programa de estabilidad actualizado de España para 2012-2015 \{COM(2012) 310 final\} (páginas 25 y 26).

[4] COMISIÓN NACIONAL DE ENERGÍA/COMISIÓN NACIONAL DE LOS MERCADOS Y COMPETENCIA (CNE/CNMC) (2002-2013): Información Estadística sobre las Ventas de Energía del Régimen Especial (2002-2013).

[5] COMISIÓN NACIONAL DE ENERGÍA (CNE) (2000-2007): Liquidaciones definitivas del sector eléctrico año 2000, 2001, 2002, 2003, 2004, 2005, 2006, 2007.

[6] COMISIÓN NACIONAL DE ENERGÍA/COMISIÓN NACIONAL DE LOS MERCADOS Y LA COMPETENCIA (CNE/CNMC) (2008-2013): Liquidaciones provisionales del sector eléctrico n. ${ }^{\circ}$ 14/2008, 14/2009, 14/2010, 14/2011, 14/2012, 14/2013.

[7] COMISIÓN NACIONAL DE LOS MERCADOS Y LA COMPETENCIA (CNMC) (2009): Informe sobre el desarrollo de la 25 subasta CESUR previsto en el artículo 14.3 de la Orden ITC/1659/2009, de 22 de junio.

[8] COMISIÓN NACIONAL DE LOS MERCADOS Y LA COMPETENCIA (CNMC) (2010-2014): Liquidación número 7 del sector eléctrico año 2010, 2011, 2012, 2013. 
[9] CNMC (2013): COMISIÓN NACIONAL DE LOS MERCADOS Y LA COMPETENCIA: Nota resumen del saldo de la deuda del sistema eléctrico a 8/11/2013.

[10] CNMC (2014): COMISIÓN NACIONAL DE LOS MERCADOS Y LA COMPETENCIA: Informe sobre la propuesta de Real Decreto por el que se establece la metodología de cálculo de los precios voluntarios para el pequeño consumidor y su régimen de contratación, 25 de febrero de 2014.

[11] DE LOS LLANOS MATEA, M. (2013): «El fondo de titulización del déficit del sistema eléctrico», Boletín Económico de ICE, nñumero 3039, pp. 15-23.

[12] DIRECTIVA 2003/54/CE DEL PARLAMENTO EUROPEO Y DEL CONSEJO de 26 de junio de 2003 sobre normas comunes para el mercado interior de la electricidad y por la que se deroga la Directiva 96/92/CE-

[13] DIRECTIVA 2009/72/CE DEL PARLAMENTO EUROPEO Y DEL CONSEJO de 13 de julio de 2009 sobre normas comunes para el mercado interior de la electricidad y por la que se deroga la Directiva 2003/54/CE.

[14] Ley 54/1997, de 27 de noviembre, del sector eléctrico. BOE número 285, pp 3509735126

[15] LEY 15/2012, de 27 de diciembre, de medidas fiscales para la sostenibilidad energética. BOE n. ${ }^{\circ} 312$, pp 88081-88096.

[16] LEY 24/2013, de 26 de diciembre, del Sector Eléctrico 2013. BOE n. . 310, pp 105198105294.

[17] ORDEN IET/346/2014, de 7 de marzo, por la que se modifica la Orden IET/2013/2013, de 31 de octubre, por la que se regula el mecanismo competitivo de asignación del servicio de gestión de la demanda de interrumpibilidad.

[18] ORDEN IET/1045/2014, de 16 de junio, por la que se aprueban los parámetros retributivos de las instalaciones tipo aplicables a determinadas instalaciones de producción de energía eléctrica a partir de fuentes de energía renovables, cogeneración y residuos.. BOE n. ${ }^{\circ} 150$, pp 46430-48175.

[19] ORDEN ITC/3993/2006, de 29 de diciembre, por la que se establece la retribución de determinadas actividades reguladas del sector gasista.

[20] ORDEN ITC/400/2007, de 26 de febrero, por la que se regulan los contratos bilaterales que firmen las empresas distribuidoras para el suministro a tarifa en el territorio peninsular.

[21] ORDEN ITC/1659/2009, de 22 de junio, por la que se establece el mecanismo de traspaso de clientes del mercado a tarifa al suministro de último recurso de energía eléctrica y el procedimiento de cálculo y estructura de las tarifas de último recurso de energía eléctrica.

[22] ORDEN ITC/1601/2010, de 11 de junio, por la que se regulan las subastas CESUR a que se refiere la Orden ITC/1659/2009, de 22 de junio, a los efectos de la determinación del coste estimado de los contratos mayoristas para el cálculo de la tarifa de último recurso.

[23] REAL DECRETO-LEY 3/2006, de 24 de febrero, por el que se modifica el mecanismo de casación de las ofertas de venta y adquisición de energía presentadas simultáneamente al mercado diario e intradiario de producción por sujetos del sector eléctrico pertenecientes al mismo grupo empresarial.

[24] REAL DECRETO 661/2007, de 25 de mayo, por el que se regula la actividad de producción de energía eléctrica en régimen especial. BOE número 126, pp 2284622886. 
[25] REAL DECRETO-LEY 6/2009, de 30 de abril, por el que se adoptan determinadas medidas en el sector energético y se aprueba el bono social. BOE número 111, pp 3940439419.

[26] REAL DECRETO-LEY 1614/2010, de 7 de diciembre, por el que se regulan y modifican determinados aspectos relativos a la actividad de producción de energía eléctrica a partir de tecnologías solar termoeléctrica y eólica.

[27] REAL DECRETO-LEY 1/2012, de 27 de enero, por el que se procede a la suspensión de los procedimientos de preasignación de retribución y a la supresión de los incentivos económicos para nuevas instalaciones de producción de energía eléctrica a partir de cogeneración, fuentes de energía renovables y residuos. BOE número 24, pp 80688072.

[28] REAL DECRETO-LEY 2/2013, de 1 de febrero, de medidas urgentes en el sistema eléctrico y en el sector financiero. BOE número 29, pp 9072-9077.

[29] REAL DECRETO-LEY 9/2013, de 12 de julio, por el que se adoptan medidas urgentes para garantizar la estabilidad financiera del sistema eléctrico. BOE número 167, pp 52106-52147.

[30] REAL DECRETO 216/2014, de 28 de marzo, por el que se establece la metodología de cálculo de los precios voluntarios para el pequeño consumidor de energía eléctrica y su régimen jurídico de contratación.

[31] REAL DECRETO 413/2014, de 6 de junio, regula la actividad de producción eléctrica mediante energías renovables, cogeneración y residuos.

[32] RESOLUCIÓN de 9 de mayo de 2014, de la Secretaría de Estado de Energía, por la que se aprueban las Reglas de funcionamiento de los mercados diario e intradiario de producción de energía eléctrica y el contrato de adhesión a dichas reglas. 